\title{
Arthrogryposis multiplex congenita
}

INSERM

\section{Source}

INSERM. (1999). Orphanet: an online rare disease and orphan drug data base.

Arthrogryposis multiplex congenita. ORPHA:1037

Arthrogryposis multiplex congenita (AMC) is a group of disorders characterized by congenital limb contractures. It manifests as limitation of movement of multiple limb joints at birth that is usually non-progressive and may include muscle weakness and fibrosis. AMC is always associated with decreased intrauterine fetal movement which leads secondarily to the contractures. 